\title{
pl6 gene transfer increases cell killing with abnormal nucleation after ionising radiation in glioma cells
}

\author{
S Hama*, , S Matsuura ${ }^{2}$, H Tauchi $^{2}$, F Yamasaki', Y Kajiwara', K Arita', H Yoshioka', Y Heike ${ }^{3}$, K Mandai $^{4}$ \\ and K Kurisu' \\ 'Department of Neurosurgery, Hiroshima University School of Medicine, Kasumi I-2-3, Minami-ku, Hiroshima 734-855 I, Japan; ${ }^{2}$ Department of \\ Radiation Biology, Research Institute for Radiation Biology and Medicine, Hiroshima University, Kasumi I-2-3, Minami-ku, Hiroshima 734-8553, Japan; \\ ${ }^{3}$ Division of Clinical Research, National Shikoku Cancer Center Hospital, Horinouchi 13, Matsuyama, Ehime 790-0007, Japan; ${ }^{4}$ Division of Pathology, \\ National Shikoku Cancer Center Hospital, Horinouchi I3, Matsuyama, Ehime 790-0007, Japan
}

It is well established that cells synchronised at the GI -S phase are highly radiosensitive. In this study, p I6-null human glioma cell lines were induced into $\mathrm{Gl}$ cell cycle arrest by adenovirus-mediated pl6 gene transfer, and examined for radiation-induced cell killing. Clonogenic analysis and trypan blue extraction test showed that the p 16 gene transfer enhanced radiation-induced cell killing in $\mathrm{p} / 6$ null glioma cell lines. TUNEL assays and pulse-field gel electrophoresis confirmed that the radiation-induced cell killing of pl6transfected cells could be caused by a nonapoptotic mechanism. Gimsa staining demonstrated that irradiation alone or Ax-mock infection plus irradiation results in a slight increase in the frequency of cells with abnormal nucleus, compared to unirradiated uninfected or Ax-mock infected cells. However, Ax-hpl 6 or Ax-hp2I infection alone modestly increased the frequency of cells with abnormal nucleus (especially bi- and multinucleation), and 4-Gy irradiation of Ax-hpl6 or Ax-hp2I infected cells substantially enhanced this frequency. These results suggest that there exists some unknown interaction between radiation and pl 6 in cytoplasm/ membranes, which decreases cytokinesis and promotes abnormal nucleation. Thus, pl6 expression prevented radiation-induced apoptosis by promoting abnormal nucleation, thereby leading to another mode of cell death. British Journal of Cancer (2003) 89, | 802- |8| I. doi:I0.1038/sj.bjc.660 I299 www.bjcancer.com (c) 2003 Cancer Research UK

Keywords: pl6; ionising radiation; abnormal nucleation; apoptosis; human glioma cell line

Human malignant gliomas diffusely invade surrounding normal brain tissue. Surgical removal of the entire tumour is difficult, and radiation therapy is often administered as an adjuvant in the treatment of incompletely resected tumours. However, the clinical effectiveness of irradiation has been limited. In almost all cases, tumours are refractory to current treatments, and the patient dies from brain herniation due to unrestrained growth of the tumour (Giangaspero and Burger, 1983). The median survival time of malignant glioma patients is less than 2 years, despite extensive and multidisciplinary treatment.

Glioma cells have been reported to display abnormality in one or more genes responsible for regulating cell-cycle progression. Such genes include the well-characterised tumour suppressor genes p53, pRb, cyclin D, p21 and p16 (Gomez-Manzano et al, 1997; Costanzi-Strauss et al, 1998; Buschges et al, 1999). p53 inhibits cell-cycle progression in response to various cellular stresses by direct transactivation of p21, an important mediator of cell proliferation, differentiation and senescence (el-Deiry et al, 1993; Xiong et al, 1993; Noda et al, 1994). p21 inhibits the ability of cyclin-dependent kinases (CDKs) to phosphorylate $\mathrm{pRb}$, thereby inhibiting cell entry into the S phase. Similarly, p16 inhibits the ability of CDK4 and CDK6 to regulate the phosphorylation status of $\mathrm{pRb}$, thereby also controlling progression into the $\mathrm{S}$ phase

*Correspondence: Dr S Hama; E-mail: ml-hns@hiroshima-u.ac.jp Received 16 June 2003; revised 31 July 2003; accepted 5 August 2003
(Nobori et al, 1994; Lukas et al, 1995). Recently, several authors have shown that $4-52 \%$ of clinical samples of malignant glioma and $68-87 \%$ of glioma cell lines have a homozygous deletion of the p16 locus (He et al, 1994; Nobori et al, 1994; Schmidt et al, 1994; Ueki et al, 1996; Hama et al, 1997). Therefore, restoration of normal function of both p53 and p16 by gene therapy is an attractive strategy for glioma treatment.

Adenovirus-mediated gene transfer of wild-type p16 following irradiation has previously been shown (by clonogenic survival assay) to enhance radiosensitivity in lung non-small-cell cancer cells (Kawabe et al, 2000). The results of clonogenic survival assays clearly indicate that cells synchronised at the late $S$ and early G1 phase are the most radioresistant, whereas cells synchronized at the $M$ phase or at the late G1 to early $S$ phase are the most radiosensitive (Terasima and Tolmach, 1963; Okada, 1970). The regulatory cascade comprising pRb, cyclin D1, CDK4/CDK6 and p16 plays a central role in cell-cycle control, and may govern radiosensitivity as well.

We have previously demonstrated that p16 gene transfer into human glioma cells using an adenovirus vector constructed with the COS-TPC method completely inhibits proliferation of the cells via $\mathrm{G} 1$ arrest for over 5 days (Hama et al, 1998). This adenovirus vector has a high transfection efficiency and provides stable, longterm gene expression. The purpose of this study was to determine whether adenovirus-mediated p16 gene transfer is a suitable gene replacement therapy for human glioma. 


\section{MATERIALS AND METHODS}

\section{Cells}

U251MG cells (human glioma; p16, homozygous deletion; p21, undetectable protein expression; p53, mutated at codon 273 [CGT/ CAT Arg/His]) and D54MG cells (human glioma; p16, undetectable protein expression; p53, wild type) were used in this study (Fueyo et al, 1996; Gomez-Manzano et al, 1996; Hama et al, 1998; Kawada et al, 1998). U251MG cells were obtained from the Japanese Cancer Research Resources Bank (Tokyo, Japan). D54MG cells were provided by Dr DT Curiel (Miller et al, 1998).

\section{Adenovirus vectors}

The p16- or p21-expressing adenovirus vectors used here were also used in previous studies of ours (Hama et al, 1998; Yamasaki et al, 2003). In brief, replication-defective adenovirus vectors were based on the human adenovirus type 5(Ad5) serotype. The full-length human p16 cDNA was a gift from Dr T Nobori, and the full-length p21 cDNA was purchased from the Meiji Institute of Health (Nobori et al, 1994; Noda et al, 1994). Recombinant adenovirus vectors containing the full-length human p16 gene (Ax-hp16) or p21 gene (Ax-hp21) were generated using the COS-TPC method, as described elsewhere (Niwa et al, 1991; Miyake et al, 1996). Exogenic p16 or p21 gene expression after Ax-hp16 or Ax-hp21 infection has been achieved in previous studies (Hama et al, 1998; Yamasaki et al, 2003). Control vectors consisted of recombinant adenoviruses containing an expression cassette without any genome (mock adenovirus vector, Ax-mock). The optimal conditions of the virus infection of each cell line for adequate gene transfer were evaluated as described elsewhere (Hama et al, 1998).

For transient infection, the culture medium was replaced $2 \mathrm{~h}$ after infection with the adenovirus vector; for continuous infection, the medium was not changed until analysis. Adenovirus expression appeared to continue for 3-5 days after transient infection, and for over 5-7 days during continuous infection (Hama et al, 1998). In the present study, most gene transfer was performed using continuous infection.

\section{Irradiation and adenovirus infection}

To obtain uniform and reproducible irradiation conditions, cells were trypsinised, counted, placed in the glass tube, and irradiated using a ${ }^{60} \mathrm{Co}$ source at a dose rate of $0.65 \mathrm{~Gy} \mathrm{~min}^{-1}$, as described elsewhere (Tauchi et al, 2001). Known numbers of cells were then replated in culture dishes and returned to the incubator. One day after irradiation, U251MG cells were infected with adenovirus vector at 5 multiplicity of infection (MOI), and D54MG cells were infected at 10 MOI.

\section{Cell survival and cell viability analysis}

Cell survival was assessed using clonogenic assays in monolayer culture, as described elsewhere (Tauchi et al, 2001). In brief, the cells were trypsinised, counted, placed in the glass tube and irradiated, as described above. Known numbers of cells were then replated in 100-mm culture dishes and returned to the incubator to allow macroscopic colony development. The surviving fractions following a given treatment were calculated based on survival of nonirradiated noninfected cells.

The viability of cells with or without irradiation $(4 \mathrm{~Gy})$ was analysed using trypan blue exclusion, as described elsewhere (Hama et al, 1998). Each experiment was repeated at least twice.

\section{Analysis of cell cycle by flow cytometry}

Cell-cycle of U251MG cells and D54MG cells was assessed using flow cytometry, as described elsewhere (Hama et al, 1998). In short, these cells were plated in a 100-mm dish and infected with Ax-hp16 or Ax-mock after irradiation. The cells were collected by trypsinisation after 3 or 6 days of incubation, washed twice with PBS, fixed with $75 \%$ ethanol and maintained at $4{ }^{\circ} \mathrm{C}$ for $48 \mathrm{~h}$. After centrifugation at $1500 \mathrm{rpm}$ for $10 \mathrm{~min}$, the cells were washed with PBS and resuspended in $1 \mathrm{ml}$ of lysis buffer $(0.1 \%$ Triton-X 100 , $0.1 \%$ RNaseA) at $4{ }^{\circ} \mathrm{C}$ overnight to free their nuclei. Immediately before analysis, $1 \mathrm{ml}$ of $50 \mu \mathrm{g} \mathrm{ml}^{-1}$ propidium iodide (PI) solution (final concentration, $25 \mu \mathrm{g} \mathrm{ml}^{-1}$ ) was added to the cell samples. Propidium iodide fluorescence of individual nuclei was measured using a FACscan (Becton Dickinson Co., Lincoln Park, NJ, USA). Data were analysed with the ModFit LT program (Becton Dickinson Co., Lincoln Park, NJ, USA). Each experiment was repeated at least twice.

\section{Detection of apoptosis}

Radiation-induced apoptosis was assessed morphologically by TUNEL assay using the In Situ Apoptosis Detection Kit (Cat. MK500, Takara Bio Inc., Ootsu, Japan). Cells were irradiated with a dose of $4 \mathrm{~Gy}$, and were infected with Ax-mock, Ax-hp16 or Ax-p21 as described above. These cells were cultured in chamber slides for further 2 or 6 days. Then, after fixation with $4 \%$ formaldehyde, they were stained according to the manufacturer's instructions, and photographs were taken under $160 \times$ magnification, using a Nikon OPTIPHOT-2 fluorescence microscope. The apoptotic index (AI) was defined as the percentage of TUNEL-positive cells in a $200 \times$ magnified field, and the AI values of the experimental groups were compared. Two different people blinded to the treatment counted the positive cells in three microscopic fields on one slide from each specimen; there were no significant differences between the counts they obtained. Each experiment was repeated at least twice.

\section{Pulse-field gel electrophoresis (PFGE)}

Induction of cleavage of chromatin in U251MG cells was determined by PFGE, as described elsewhere (Hwang et al, 2000; Cipriani et al, 2001). In breif, U251MG cells were infected with Axhp16 or Ax-mock after irradiation and collected by trypsinisation after 5 days of incubation. Cell pellets containing $2 \times 10^{7}$ U251MG cells/sample were resuspended in $1000 \mu \mathrm{l}$ of $\mathrm{Ca}^{2+}$ $\mathrm{Mg}^{2+}$-free PBS and $1000 \mu \mathrm{l}$ of $1 \%$ low-melt agarose, and solidified at $4{ }^{\circ} \mathrm{C}$ for $30 \mathrm{~min}$. Plugs were digested for $48 \mathrm{~h}$ at $55^{\circ} \mathrm{C}$ in $5 \mathrm{ml}$ of digestion buffer containing $100 \mathrm{~mm}$ EDTA ( $\mathrm{pH} \mathrm{8.0),0.2 \%} \mathrm{sodium}$ deoxycholate, $1 \%$ sodium lauryl sarcosine, and $1 \mathrm{mg} \mathrm{ml}^{-1}$ proteinase $\mathrm{K}$. The plugs were then washed four times in washing buffer (20 mM Tris- $\mathrm{HCl}(\mathrm{pH} 8)$ and $50 \mathrm{~mm}$ EDTA), loaded into a $1.2 \%$ agarose gel, and run in $0.5 \mathrm{x}$ TBE buffer for $22 \mathrm{~h}$ using the CHEFDR II electrophoresis cell (Bio-Rad, Hercules, CA, USA). Running conditions were optimised to separate $50-1000-\mathrm{kbp}$ DNA fragments, as follows: temperature, $14^{\circ} \mathrm{C}$; switch time, $50-90 \mathrm{~s}$; angle, $120^{\circ}$; voltage gradient, $6 \mathrm{~V} \mathrm{~cm}^{-1}$. The standard-size DNA ladder was included. After staining with $0.5 \mu \mathrm{g} \mathrm{ml}^{-1}$ ethidium bromide, bands were visualised using a UV transilluminator (254$360 \mathrm{~nm})$.

\section{Evaluation of nuclear morphology}

Nuclear morphologic changes in U251MG or D54MG cells were evaluated using Giemsa staining, as described elsewhere (Bhattathiri et al, 1998a, b; Bhattathiri, 2001). U251MG and D54MG cells were irradiated with a dose of $4 \mathrm{~Gy}$, and were infected with Axmock, Ax-hp16 or Ax-p21 as described above. These cells were cultured for a further 6 days, collected by trypsinisation, spread on a clean glass slide, fixed in ethyl alcohol and stained with Giemsa stain. The frequency of micronucleated, nuclear budded, binucleated and multinucleated tumour cells was evaluated. The 
criteria for identification of these cells are described elsewhere (Bhattathiri et al, 1998a, b; Bhattathiri, 2001). Briefly, micronucleated cells contain small round bodies of nuclear material within the cytoplasm, separate from the main nucleus (the cells indicated with one arrow in Figure 4D and E). Nuclear budded cells have buds adjoining the main nucleus; these could be micronuclei, if not for the fact that their separation from the main nucleus is indistinct (the cells indicated with two arrows in Figure 4D and E). Binucleated cells have two nuclei. Multinucleated cells have more than two nuclei (Figure 4C and the cells indicated with three arrows in Figure 4E). Around 500-1000 cells were evaluated from the samples collected on each occasion, and the results were expressed in comparison with 1000 culture cells with a single nucleus (ie, uninucleated cells). This experiment was repeated at least twice.

\section{Statistical analysis}

Statistical analysis was performed by Student's $t$-test using Stat View 5.0 (Abacus Concepts, Inc). A $P$-value of $<0.05$ was considered to indicate statistical significance.

\section{RESULTS}

\section{Effect of p16 gene transfer on radiation-induced cell killing}

We first tested whether Ax-hp16 infection increased the radiosensitivity of human glioma cell lines in vitro. Clonogenic assays were performed on two glioma cell lines: U251MG (p16-null, p21null and p53-mutant) and D54MG (p16-null and p53-wild type). These cell lines were infected with either Ax-hp16 or Ax-mock after irradiation. In preliminary experiments, we compared the effect on radiosensitivity between various time courses of irradiation and infection (transient infection $v s$ continuous infection). The maximum effect was obtained when cells were continuously infected with Ax-hp16 after irradiation. We also assessed continuous adenovirus infection before irradiation; however, this procedure required collection of cells by trypsinisation after infection, transfer of the medium plus cells and adenovirus vectors to a glass tube, irradiation and replating of irradiated cells on the culture dishes without exchanging the medium (containing the adenovirus vector). This procedure was intricate and not reproducible. Therefore, we chose to irradiate cells prior to adenoviral infection. As continuous adenovirus infection is particularly likely to cause direct cytotoxicity, we assessed the cytotoxicity of the vector alone using plating efficiency as the end point. Cytotoxicity became evident at MOI values between 5 and 10 in U251MG cells, and between 7.5 and 15 in D54MG cells. Then, in further preliminary experiments, we used MOI values of 5 and 10 in U251MG cells and MOI values of 7.5, 10 and 15 in D54MG cells to assess radiosensitisation (data not shown). For U251MG, the most effective radiosensitisation was achieved using an MOI value of 5; for D54MG, this MOI value was 10 .

As shown in Figure 1A, the survival of nonirradiated U251MG cells was slightly reduced by Ax-hp16 infection to $83 \%$; at $2 \mathrm{~Gy}$, it was markedly reduced from 77 to $0.69 \%$; at $4 \mathrm{~Gy}$, it was reduced from 15 to $0.019 \%$. The percent survival of nonirradiated D54MG cells was slightly reduced by Ax-hp16 infection to $55 \%$; at $2 \mathrm{~Gy}$, it was markedly reduced from 31 to $9.1 \%$; at $4 \mathrm{~Gy}$, it was reduced from 8.5 to $0.67 \%$ (Figure $1 \mathrm{~B}$ ). Ax-mock infection alone did not produce marked sensitisation in either of the two cell lines in the range of 2-6Gy. U251MG cells or D54MG cells infected with Ax-hp16 were significantly more radiosensitive than noninfected or Ax-mock-infected cells. This trend was similar to that of Ax-hp21-infected U251MG cells (Figure 1C).
Next, we examined the cell viability of the U251MG and D54MG cells using the trypan blue exclusion test (Figure 1D, E). Until 5 days after irradiation, there was no difference in viability between Ax-h-p16-infected cells and Ax-mock-infected or noninfected cells. In contrast, 7-8 days after irradiation, the viability of irradiated Ax-hp16-infected cells gradually decreased, compared with Axmock-infected, noninfected and nonirradiated cells. The viability of nonirradiated Ax-hp16-infected U251MG cells decreased gradually. The viability of irradiated Ax-hp16-infected U251MG cells decreased more significantly than that of nonirradiated Axhp16-infected cells $(P=0.005$ on day 11 , and $P=0.031$ on day 13$)$.

Thus, adenovirus-mediated p16 gene transfer enhanced the radiosensitivity of glioma cell lines that lack p16, but contain either wt-p53 or mut-p53.

\section{Effect of p16 gene transfer on cell cycle of U251MG cells after irradiation}

To determine whether Ax-hp16 infection induced G1 cell-cycle arrest in U251MG or D54MG cells, cell-cycle status was analysed on days 3 and 6 using flow cytometry. Figure 2 shows the histogram of cell-cycle status following irradiation and/or infection with Ax-mock or Ax-hp16 on day 6 (the data for day 3 were almost the same as that of day 6 , and is not shown).

Nonirradiated Ax-hp16-infected U251MG and D54MG cells showed G1 arrest during the 6 days following infection, whereas the cell-cycle profile of Ax-mock-infected cells was the same as that of noninfected cells. In U251MG cells, after 4-Gy irradiation, G2/M accumulation occurred in Ax-mock-infected and noninfected cells. G2/M accumulation was markedly decreased in Ax-hp16-infected cells, compared with Ax-mock-infected and noninfected cells. In D54MG cells, after 4-Gy irradiation, G1 accumulation occurred in Ax-mock-infected and noninfected cells, and was significantly increased in Ax-hp16-infected cells. These results demonstrate that Ax-hp16 infection resulted in G1 arrest.

\section{Apoptotic analysis}

To determine whether the death of Ax-hp16-infected U251MG cells after irradiation was due to apoptosis, we performed the TUNEL assay and measured the level of apoptosis on the basis of TUNEL positivity following various treatments 3 and 7 days after irradiation. Figure $3 \mathrm{~A}$ shows the percentage of TUNEL-positive cells among U251MG cells that were noninfected, Ax-mockinfected, Ax-hp16-infected or Ax-hp21-infected, with or without irradiation. Irradiated cells that were noninfected or Ax-mockinfected had a higher proportion of TUNEL-positive cells (uninfected cells, $22.0 \%$ on day 3 and $15.1 \%$ on day 7 ; Ax-mock infected cells, $10.6 \%$ on day 3 and $12.2 \%$ on day 7) than the corresponding unirradiated cells. Irradiated cells that were Axhp16- or Ax-hp21-infected had the same proportion of TUNELpositive cells (Ax-hp16-infected cells, $2.1 \%$ on day 3 and $0.7 \%$ on day 7; Ax-hp21-infected cells, $3.6 \%$ on day 3 and $3.1 \%$ on day 7 ) as the corresponding unirradiated cells. D54MG showed a similar trend (irradiation alone, $8.0 \%$ on day 3 and $7.4 \%$ on day $7 ; \mathrm{Ax}$ mock infection plus irradiation, $12.0 \%$ on day 3 and $9.0 \%$ on day 7 ; Ax-hp16 infection plus irradiation, $4.4 \%$ on day 3 and $1.3 \%$ on day 7) (Figure 3B).

As shown in Figure 3C, irradiated noninfected or Ax-mockinfected U251MG cells were identified as scattered clusters of fluorescent staining, suggesting that the death of these cells was due to apoptosis. In contrast, Ax-hp16-infected U251MG cells did not emit fluorescent signals even after irradiation. p21 transfection caused a similar fluorescent staining in irradiated cells (Figure 3C). Therefore, it is unlikely that the cell death of irradiated Ax-hp16or Ax-hp21-infected U251MG cells during the first 7 days after irradiation was due to apoptosis. 

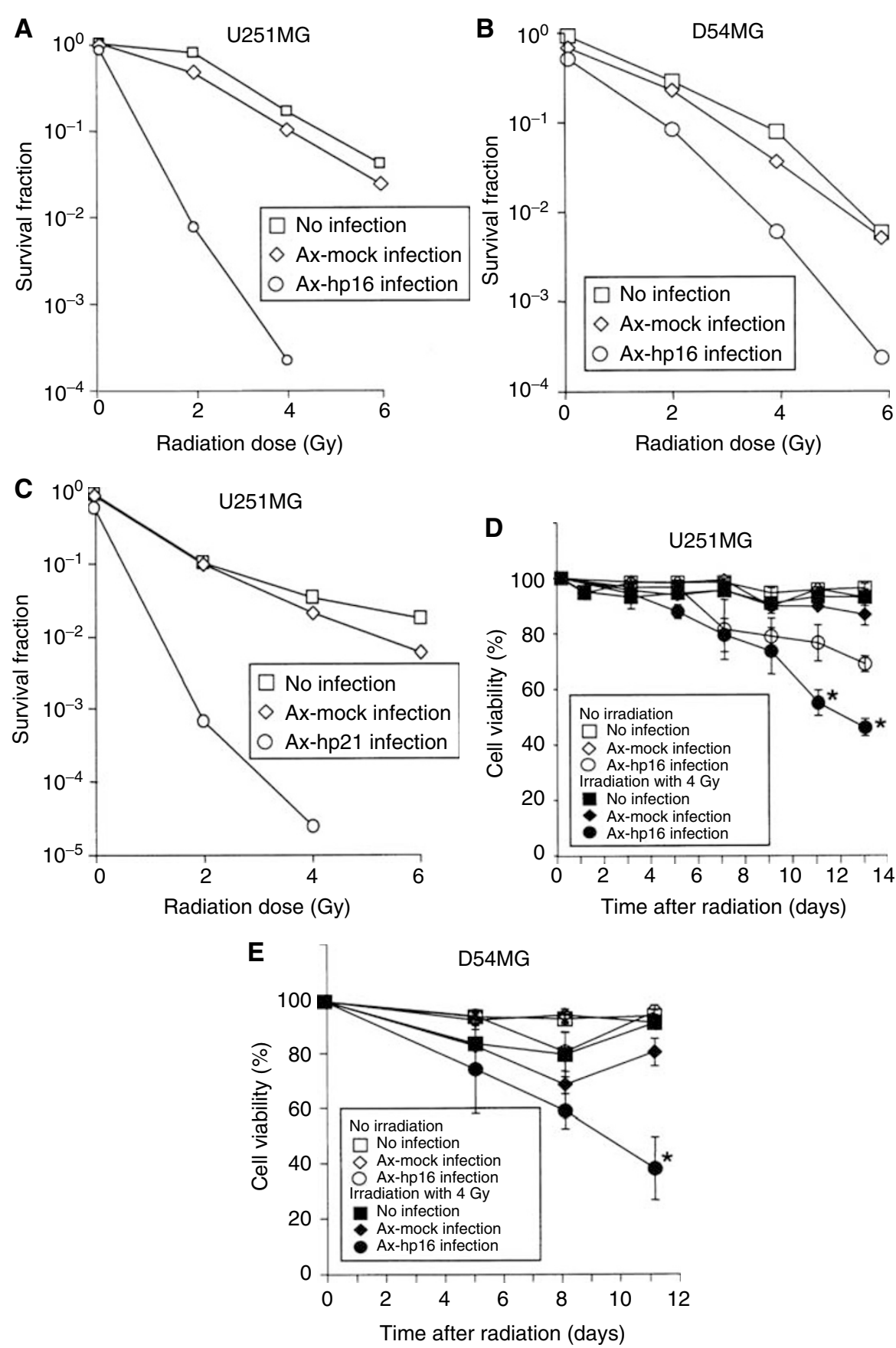

Figure I Effect of Ax-hp 16 or Ax-hp2I infection on cell survival or cell viability in p I6- or p2 I-null human glioma cell lines with or without irradiation. At I day after irradiation, U25IMG cells (pI6-null, p2I-null, p53-mut) were infected with adenovirus vector at 5 MOI, and D54MG cells (pI6-null, p53-wild) were infected at 10 MOI. (A) U25 IMG, Ax-mock or Ax-hpl 6 infection; (B) D54MG, Ax-mock or Ax-hp I6 infection; and (C) U25 IMG, Ax-mock or Axhp2I infection show cell survival determined by clonogenic assays. (D) U25IMG, Ax-mock or Ax-hp I6 infection and (E) D54MG, Ax-mock or Ax-hp I6 infection show cell viability determined using Trypan blue exclusion. The results of cell viability assays are shown as the mean number of cells at each point, with standard deviation of three wells. Statistical significance: $* P<0.05$, compared with cells other than irradiated Ax-hp I6-infected cells.

To confirm these interpretations, the chromatin structure was analysed using PFGE (Figure 3D). We investigated the cleavage of chromatin by examining the formation of 50/300-kb fragments, which are generated during the early stages of apoptosis and can be identified by PFGE (Khodarev et al, 1998; Hwang et al, 2000; Cipriani et al, 2001). The 50/300-kb fragments were formed in irradiated noninfected and Ax-mock-infected U251MG cells, but not in nonirradiated U251MG cells. Ax-hp16-infected U251MG cells did not form $50 / 300-\mathrm{kb}$ fragments, with or without irradiation. These results support our hypothesis that cell death of Ax-hp16-infected U251MG cells following irradiation is not due to apoptosis.

\section{Nuclear morphology}

To determine the nature of the radiation-induced cell death of the Ax-hp16- and Ax-hp21-infected cells, microscopic analysis using Giemsa staining was performed to measure the level 
U251MG cells
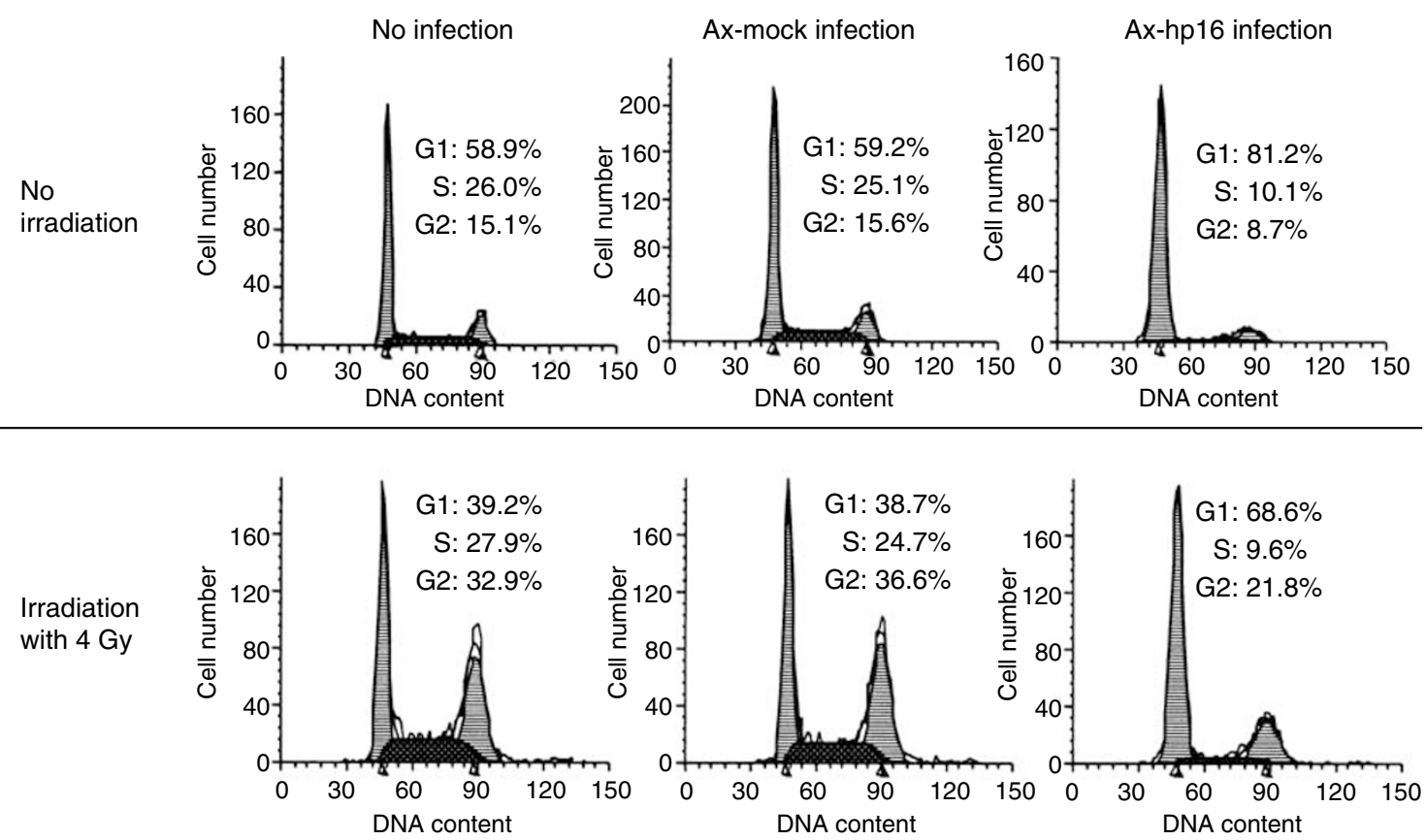

D54MG cells
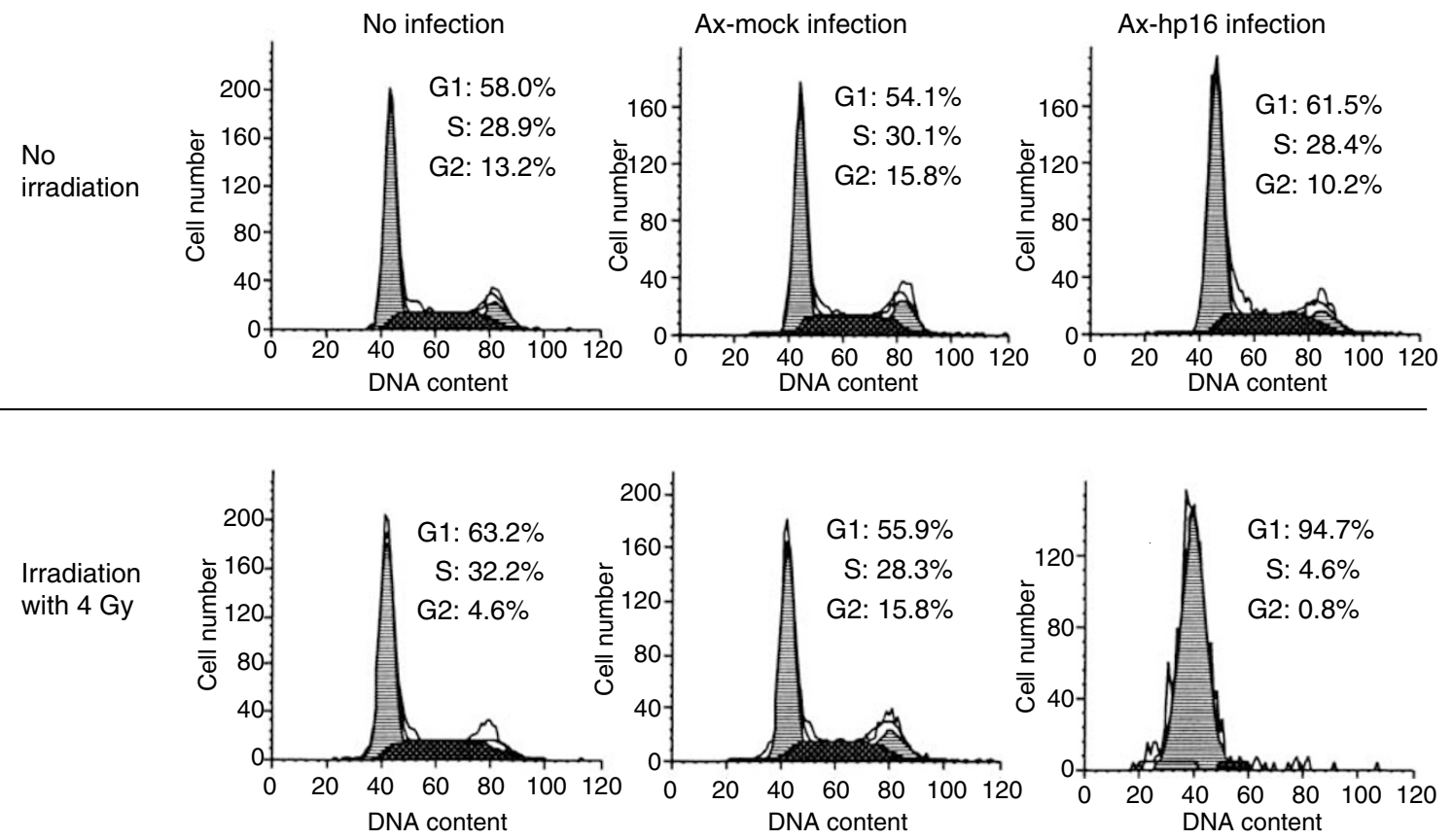

Figure 2 Effect of pl6 transduction on cell-cycle of U25IMG and D54MG cells. U25IMG (upper) and D54MG cells (lower), nonirradiated or irradiated at a dose of $4 \mathrm{~Gy}$, noninfected or infected with Ax-hp 16 or Ax-mock, were cultured for 5 days, and the cell-cycle was analysed by flow cytometry. Data are presented as a histogram, with cell number (Y-axis) plotted against DNA content (X-axis). The first peak contains the cells with diploid DNA at G0/GI. The cells in the second peak with double Pl-fluorescence intensity are tetraploid at $\mathrm{G} 2 / \mathrm{M}$. The area between the two peaks contains the cells in the $\mathrm{S}$ phase.

of abnormal nucleus changes (budding and micro-, bi- and multinucleation). The morphological appearance of these nuclear changes is shown in Figure 4C, D and E.

Figure $4 \mathrm{~A}$ shows the frequency of cells with abnormal nucleus per 1000 uninucleated cells treated with noninfection,
Ax-mock-, Ax-hp16- or Ax-hp21 infection, with or without irradiation, 7 days after irradiation. Irradiation alone and Ax-mock infection plus irradiation resulted in a slight increase in this frequency (uninfection, 64 budded, 75 micro-, 83 bi-, and 69 multinucleations; Ax-mock infection, 68 budded, 


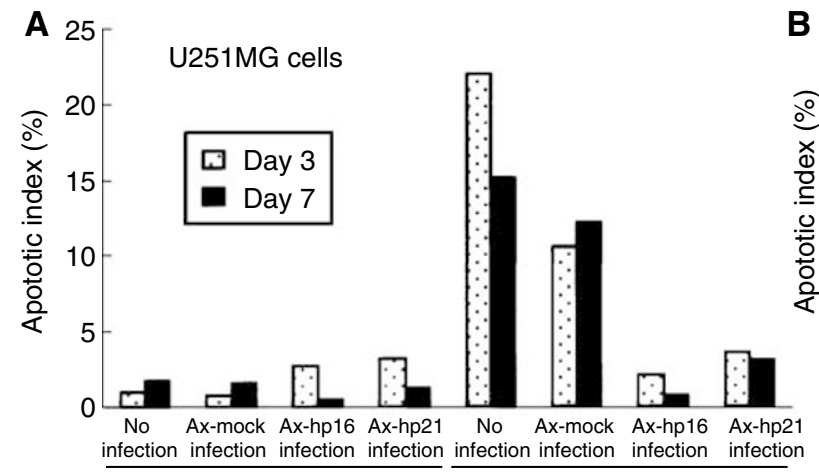

No irradiation

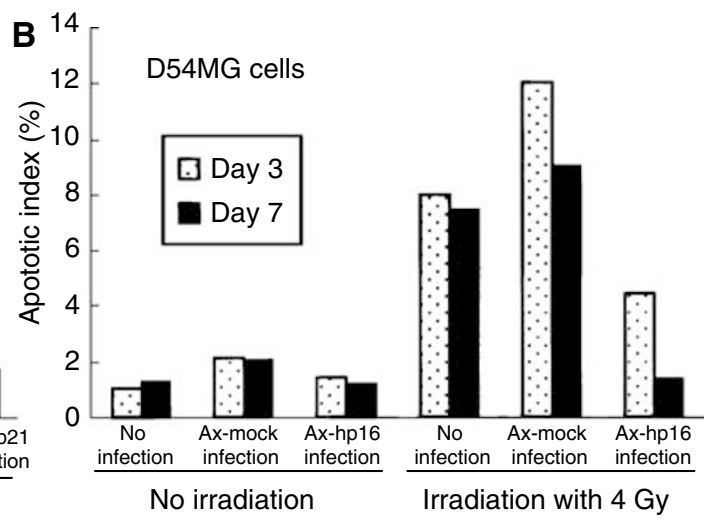

Ax-hp16 infection

Ax-hp21 infection

C No infection

Ax-mock infection
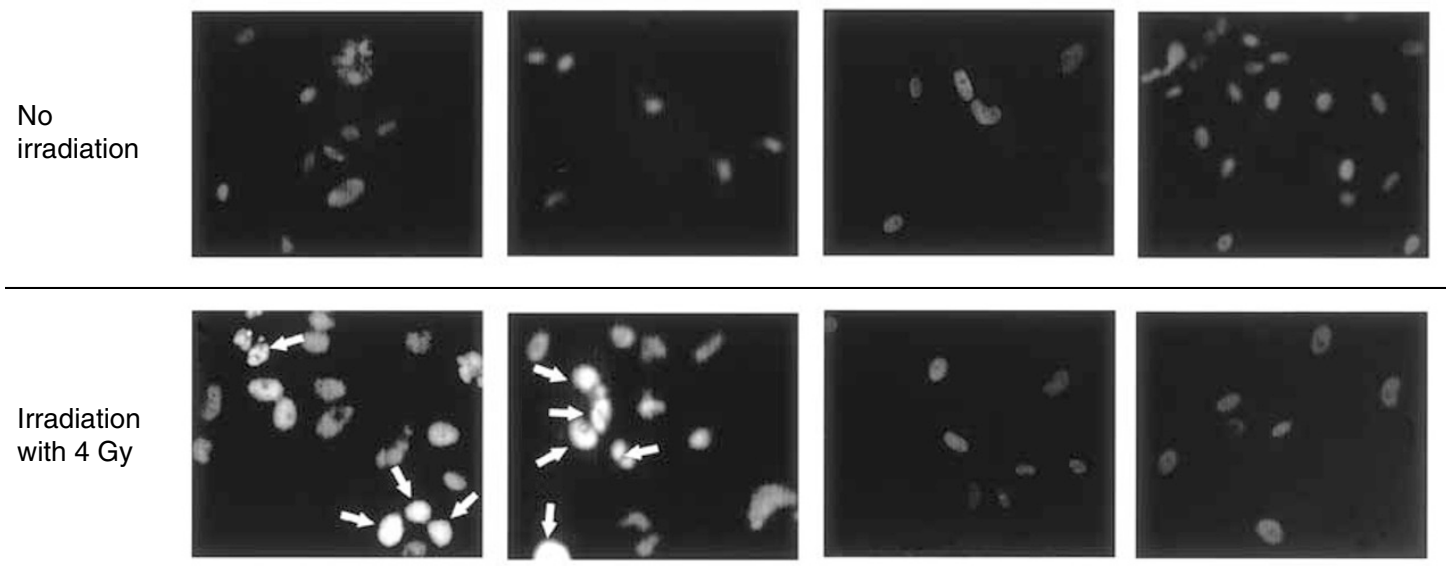

D

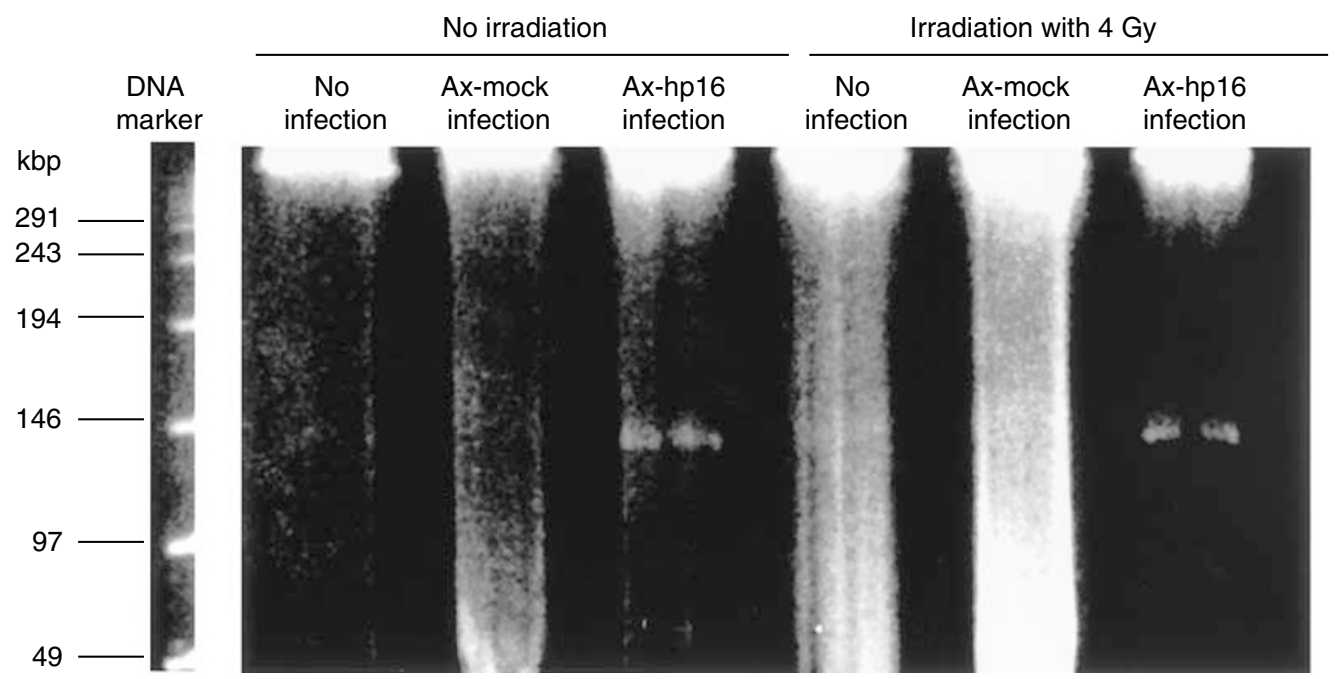

Figure 3 Apoptosis assessed by the TUNEL assay and PFGE. TUNEL assay was used for the morphological detection of apoptotic changes. U25 IMG or D54MG cells, nonirradiated or irradiated at a dose of $4 \mathrm{~Gy}$, noninfected or infected with Ax-hp I6, Ax-hp2I or Ax-mock I day after irradiation, were cultured for 2 or 6 days, and were stained according to the manufacturer's instructions after fixation with $4 \%$ formaldehyde. The Al was defined as the percentage of TUNEL-positive U25I (A) or D54MG cells (B). (C) TUNEL signals were detected by fluorescence microscopy in the nuclei of some irradiated uninfected or Ax-mock-infected U25IMG cells (arrows). (D) The chromatin structure was analysed using PFGE. U25IMG cells were treated as described in the TUNEL assay, and, 5 days after infection, cells were embedded in agarose gel and subjected to PFGE. The 50/300-kb fragments, which are generated during the early stage of apoptosis, were formed in irradiated noninfected and Ax-mock-infected U25I MG cells. The sizes of the DNA fragments are indicated on the left.

50 micro-, 54 bi-, and 100 multinucleations), compared to unirradiated uninfected or Ax-mock-infected cells. As expected, Ax-hp16 or Ax-hp21 infection alone modestly increased the frequency of cells with an abnormal nucleus, especially for bi- and multinucleation (Ax-hp16, 116 budded, 90 micro-, 207 bi-, and 90 multinucleations; Ax-hp21 infection, 97 budded, 


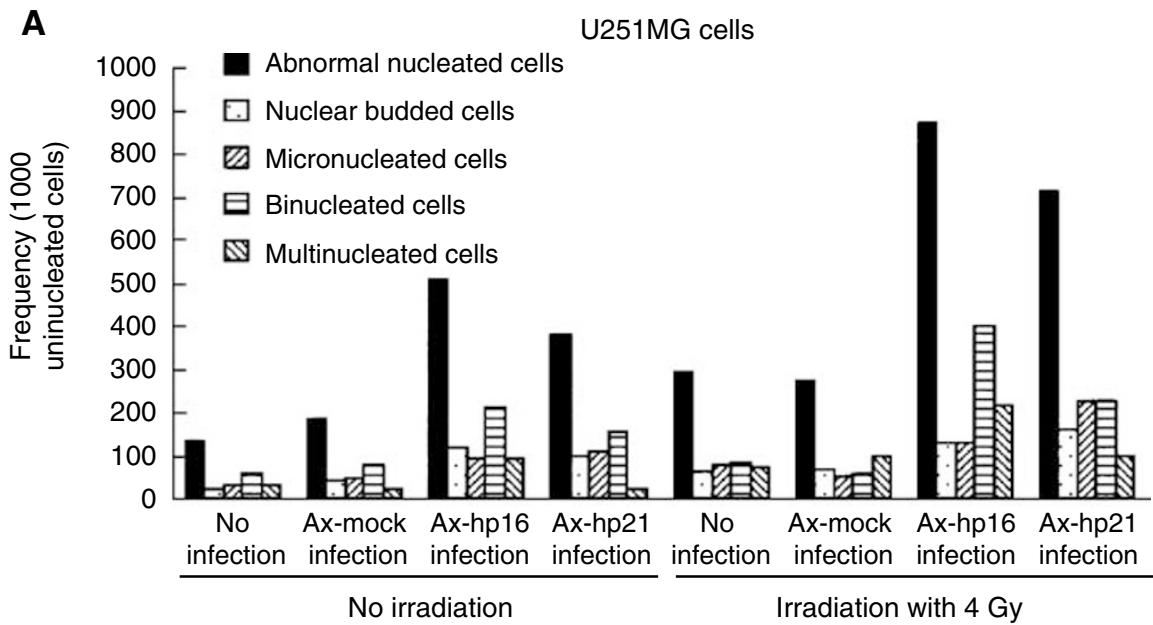

B

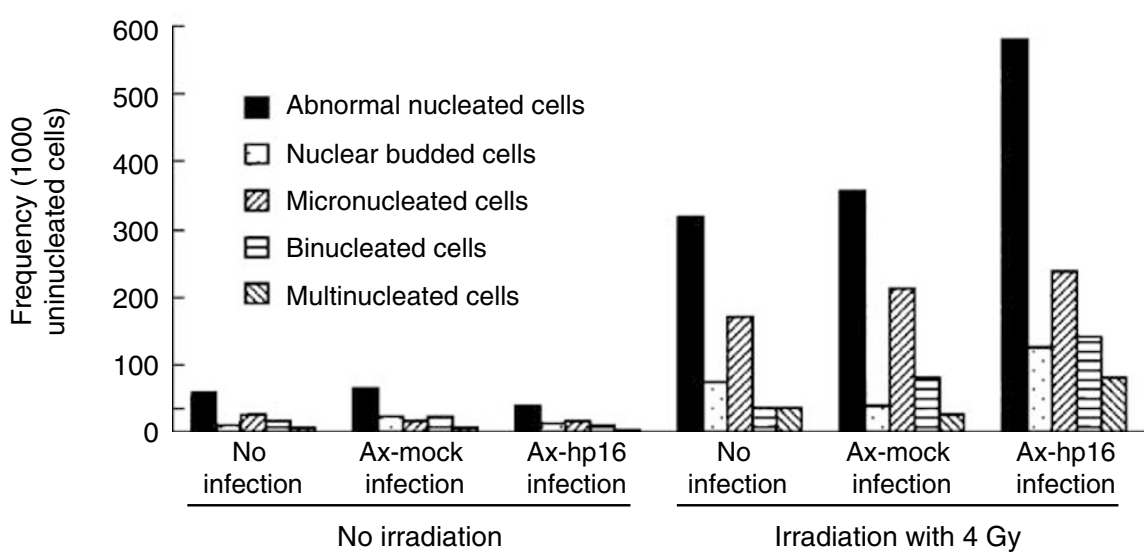

C

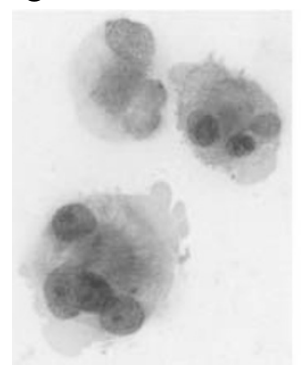

D

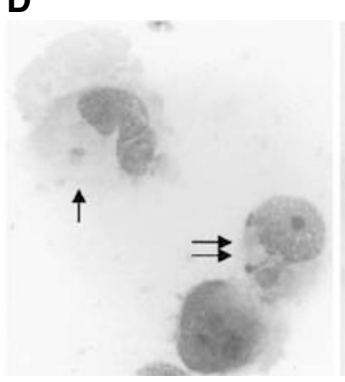

E

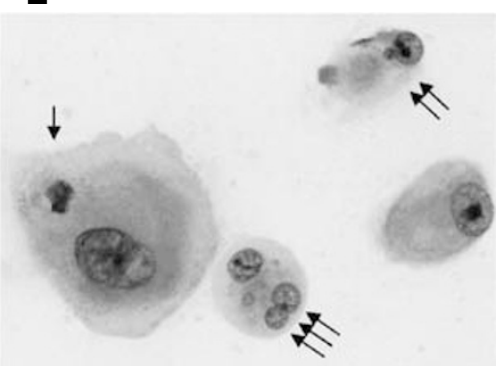

Figure 4 Effect of $\mathrm{p} 16$ or $\mathrm{p} 21$ transduction with or without irradiation on nuclear morphologic changes. U25IMG or D54MG cells were treated as described in the TUNEL assay, and nuclear morphologic changes in these treated cells were evaluated using Giemsa staining 7 days after irradiation. The frequency of abnormal nuclei (including nuclear budding, micronucleation, binucleation and multinucleation) in U25IMG (A) or D54MG cells (B) is expressed in comparison with 1000 culture cells with a single nucleus (ie, uninucleated cells). The figures show (C) multinucleation, (D) micronucleation (one arrow) and nuclear budding (double arrows) in irradiated Ax-hp I 6-infected U25 I MG cells, and (E) micronucleation (one arrow), nuclear budding (two arrows) and multinucleation (triple arrows) in irradiated Ax-hp I6-infected D54MG cells.

108 micro-, 152 bi-, and 21 multinucleations), and 4-Gy irradiation of Ax-hp16- or Ax-hp21-infected cells substantially enhanced this frequency (Ax-hp16, 128 budded, 128 micro-, 398 bi-, and 214 multinucleations; Ax-hp21 infection, 158 budded, 227 micro-, 227 bi-, and 99 multinucleations), compared with the corresponding controls (Figure 4C, D). D54MG cells showed a similar trend (Figure $4 \mathrm{~B}, \mathrm{E}$ ). These increases in the frequency of cells with an abnormal nucleus are consistent with increased abnormal nucleus formation resulting from the combined treatment of Ax-hp16 or Ax-hp21 infection plus irradiation.

\section{DISCUSSION}

The inactivation of p16 function has been linked to the development of malignant gliomas, and recent investigations have provided an insight into the manner in which this inactivation may contribute to cell proliferation (Nobori et al, 1994; Fueyo et al, 1996; Hama et al, 1997, 1998; Costanzi-Strauss et al, 1998). Previous findings indicate that p16-dependent downmodulation of $\mathrm{pRb}$ levels in the presence of overexpressed wild-type p53 causes apoptosis only in tumour cells, whereas normal cells arrest in G1 (Sandig et al, 1997). Kawabe et al (2000) 
demonstrated, by clonogenic survival assay, that adenovirusmediated p16 gene expression enhanced the radiosensitivity of non-small-cell lung cancer cells, depending on the endogenous wild-type p53 expression of the cell line. It is well established that radiation sensitivity varies with the population of the cell-cycle, and that cells at the M-phase and late G1 to the early S phase have great sensitivity (Terasima and Tolmach, 1963; Okada, 1970). Here, we assessed the ability of Ax-hp16 to enhance radiosensitivity in human glioma cell lines that lack p16, but have either wt-p53 or mut-p53.

The clonogenic survival assays indicated that the radiosensitivity of D54MG cells, which have wt-p53, was substantially increased by $\mathrm{Ax}-\mathrm{hp} 16$ infection, as reported previously (Figure 1B). However, contrary to this previous report, the radiosensitivity of U251MG cells, which do not have functional p53, was also increased by Ax-hp16 infection (Figure 1A). As indicated by trypan blue exclusion test, U251MG and D54MG cells exhibited reduced viability after irradiation in combination with p16 gene replacement (Figure 1D-E). The results indicate that Ax-hp16 infection increased cell death in a p53-independent manner.

Based on these results, we doubt that the effect of Ax-hp16 infection on radiosensitivity was induced by the direct effect of p16 or by G1 arrest. Therefore, we used a clonogenic assay to assess the effects on the radiosensitivity of an adenovirus vector expressing another G1-S regulatory factor, p21. The results show that Axhp21 had the same effect as Ax-hp16 (Figure 1C). Moreover, we examined cell-cycle status using flow cytometry (Figure 2). Radiation alone produced G1 arrest in D54MG cells and G2-M arrest in U251MG cells. The observed difference in cell-cycle progression between the two cell lines after irradiation may be due to the difference in their p53 status, and is consistent with previous findings obtained using the same cell lines (Ostruszka and Shewach, 2000). However, Ax-hp16-infected U251MG and D54MG cells were arrested in the G1 phase during the 6 days following irradiation, suggesting that the induction of G1 arrest following irradiation increases the radiosensitivity of these cells.

Kawabe et al (2000) reported that p16 gene transfer enhanced apoptotic cell death for cells that have wt-p53, but not for p53mutated or p53-deleted cells. The present findings indicate that p16 enhanced radiosensitivity via increased cell death, and that this enhancement did not depend on p53 status. We examined the underlying mechanism of this phenomenon using the TUNEL assay, and demonstrated that radiation-induced cell death of noninfected or Ax-mock-infected U251MG or D54MG cells was due to apoptosis, whereas we found that radiation-induced cell death of Ax-hp16-infected cells was not likely to be due to apoptosis. We also performed an electron microscopic assay, and detected apoptosis in noninfected U251MG cells, but not in Axhp16-infected U251 MG cells after irradiation (data not shown). The present results of PFGE and electron microscopic assay support our hypothesis that the cell death of irradiated Ax-hp16infected cells was not due to apoptosis, suggesting that a different biochemical pathway or triggering mechanisms is involved in this mode of cell death.

Questions are raised by the present observations regarding the mechanism, whereby the irradiated Ax-hp16-infected cells exhibited cell-cycle arrest at the G1 phase (as indicated by flow cytometry) and the mechanism of cell death. The cellular roles of endogenous p16 are relatively poorly understood, but it is clear that its role is not limited to G1/S arrest. In HeLa cells, G2 delay correlates with elevated p16 levels, and loss of p16 expression results in loss of UVC-induced cell-cycle delay (Wang et al, 1996). Milligan et al (1998) reported that p16 acts specifically via its binding to cdk4 to produce G2 delay responses following UVC irradiation in melanoma cell lines. Moreover, p16 is found not only in the nucleus but also in the cytoplasm, especially in cancer cells (Sano et al, 1998; Chen et al, 1999), and plays an important role in the regulation of vascular endothelial growth factor (VEGF) expression and cell senescence (Harada et al, 1999).

It has been suggested that cancer cells can escape from G1/S arrest induced by p16, RB loss and Cyclin D1 amplification, via mechanisms that can over-ride the braking effect (Sherr et al, 1996). If there is a concomitant rapid exit from G2/S arrest induced by $\mathrm{p} 16$, there is no accumulation of tetraploid cells. In malignant cells, this exit can occur by abnormal nucleation, a term that includes not only nuclear budding/micronucleation and simple unequal nuclear division but also multipolar mitosis, repeated karyokinesis with acytokinesis and all divisions not associated with normal mitosis. The formation of these structures is not detectable by flow cytometry, because this method evaluates bare nuclei (Bhattathiri, 2001). Figure 2 indicates that the distribution of values around $2 \mathrm{~N}$ and $4 \mathrm{~N}$ is wider in the Ax-hp16-infected cells (both irradiated and nonirradiated), compared to the noninfected and Ax-mock-infected cells, a finding consistent with abnormal nucleation. Therefore, we used Giemsa staining to assay the induction of micronucleation, nuclear budding, binucleation and multinucleation individually in U251MG and D54MG cells 7 days after irradiation, with or without adenovirus infection (Figure 4).

In uninfected and Ax-mock-infected cells, there was an increase in cells with the above abnormalities, especially micro- and budded nucleation. Also, in Ax-hp16- and Ax-hp21-infected cells, there was an increase in cells with the above abnormalities, especially bi- and multinucleation. In unirradiated Ax-hp16- and Ax-hp21-infected U251MG cells, there was an increase in cells with the above abnormalities, especially bi- and multinucleation, but there was little change in unirradiated D54MG cells.

The situation is likely to be different when p16 and radiation are combined, with radiation inducing rapid proliferation and p16 facilitating rapid exit from G2/S arrest by abnormal nucleation. This abolishes apoptosis induced by G2/S arrest, yet promotes cell death by abnormal nucleation. The interaction of radiation and p16 in the morphological changes of abnormal nucleation has been reported. In skin cells, increased p16 expression with cytoplasmic predominance after UV irradiation correlates with the development of bi-, multi- and micronucleated cells (Milligan et al, 1998; Pavey et al, 1999). These observations suggest an unknown interaction between radiation and p16 in cytoplasm/membranes, which decreases cytokinesis and promotes abnormal nucleation.

Fueyo et al (1996) have shown that adenovirus-mediated p16 gene transfection alone significantly inhibits cell growth, a finding not obtained in the present study; Ax-hp16-infected cells used in the present study lost cell viability, as indicated in Figure 1D. This inhibition may be due to the induction of abnormal nucleated nonclonogenic cells. Obviously, the rates of survival and formation of multinucleated cells are likely to be higher in glioblastomas (Meyer-Puttlitz et al, 1997). Abnormal nucleation can also explain why apoptosis observed with radiation alone was absent when p16 transfection and radiation were combined. Obviously, p16 expression prevented radiation-induced apoptosis by promoting abnormal nucleation, thereby leading to cell death via this alternative mechanism. The situation can be viewed as radiation enhancing the effect of p16, resulting in an increase of radiation-induced cell death.

With p21, similar effects are possible, because p21 expression has also been found to be related to multinucleation (Okahashi et al, 2001). Mutinuclear osteoclast-like cell formation from osteoblasts is related to the upregulation of $\mathrm{p} 21$, with the majority of cells remaining at the G0/G1 phase (Tanaka et al, 2000). The integrity of p21 within centrosomes is related to apoptosis, multinucleation and normal mitotic progression (Li et al, 1999). It has been reported that, in MCF-7 cells, pacllitaxel induced the accumulation of p21 in cells with G2/M DNA content; this corresponds to cells in abnormal mitosis or an interphase-like state with multiple nuclei, the increase in p21 being subsequent to mitotic arrest and associated with exit from abnormal mitosis 
leading to the formation of cells with micronuclei (Barboule et al, 1997). It is possible that radiation also affects p21 localised in the centrosome complex, resulting in bi- or multinucleation.

Cell survival (assessed by clonogenic assay) markedly decreased in irradiated Ax-hp16-infected U251MG and D54MG cells, whereas cell viability (determined by trypan blue exclusion) only slightly decreased in the same cell lines; thus, the results of these two analyses are apparently not consistent. Physically dead cells are stained by trypan blue, but the formation of cellular fragmentation or cellular debris can occur over a period of several days, depending on the type of cell death; thus, trypan blue-positive (dead) cells are thought to reflect cells that died within a few days. On the other hand, abnormal nucleated cells are thought to be clonogenically dead, but not physically dead, and can synthesise DNA and continue to divide several times before death after irradiation (Bhattathiri, 2001). Unirradiated Ax-hp16-infected U251MG cells exhibited a modest increase in the proportion of abnormally nucleated cells, and 4-Gy irradiation of Ax-hp16infected cells substantially enhanced this proportion (Figure 4A). After irradiation of Ax-hp16-infected D54MG cells, the frequency of abnormal nucleated cells increased (Figure 4B). Abnormal nucleated cells may not be completely detected by trypan blue analysis, but they are not clonogenic; thus, it is thought that the discrepancy was between cell viability and clonogenic assay.

A comparison between mock-infected and p16-infected D54MG cells (Figure 1E) shows that cell viability decreased identically for these cell populations until day 8 after irradiation. Teramoto et al (1995) examined whether E1-, E3-deleted adenovirus vectors affect the cell viability of human airway cells in primary culture, using trypan blue exclusion, and demonstrated that, as the MOI of adenovirus vectors is increased (to achieve effective gene transfer), the viability of infected cells is reduced concomitantly via apoptotic change. This reduced viability persisted for 5-7 days after vector administration. Tang et al (1997) demonstrated that irradiating cells at doses of 2-40 Gy prior to transduction could amplify recombinant adenoviral transgene products in a cell-typespecific manner. The apoptotic index of Ax-mock-infected D54MG cells increased, compared with uninfected cells, on days 3 and 7, with or without irradiation (Figure 3), but this trend was not exhibited by U251MG cells. One of the differences between U251MG and D54MG cells is p53 status; that is, p53 of U251MG is mutant, whereas that of D54MG is wild type. Therefore, it is possible that, in D54MG cells (but not in U251MG cells), irradiation amplified the recombinant adenoviral transgene products, activated a p53-dependent apoptotic pathway and increased apoptotic cell death, resulting in the reduction of cell viability, especially during the first 8 days after infection.

\section{REFERENCES}

Barboule N, Chadebech P, Baldin V, Vidal S, Valette A (1997) Involvement of p21 in mitotic exit after paclitaxel treatment in MCF-7 breast adenocarcinoma cell line. Oncogene 15: $2867-2875$

Bhattathiri NV, Bindu L, Remani P, Chandralekha B, Nair KM (1998a) Radiation-induced acute immediate nuclear abnormalities in oral cancer cells: serial cytologic evaluation. Acta Cytol 42: 1084-1090

Bhattathiri NV, Bharathykkutty C, Prathapan R, Chirayathmanjiyil DA, Nair KM (1998b) Prediction of radiosensitivity of oral cancers by serial cytological assay of nuclear changes. Radiother Oncol 49: $61-65$

Bhattathiri VN (2001) Amitotic cell divisions and tumour growth: an alternative model for cell kinetic compartments in solid tumours. Oral Oncol 37: 288-295

Buschges R, Weber RG, Actor B, Lichter P, Collins VP, Reifenberger G (1999) Amplification and expression of cyclin D genes (CCND1, CCND2 and CCND3) in human malignant gliomas. Brain Pathol 9: 435-442

Chen Q, Luo G, Li B, Samaranayake LP (1999) Expression of p16 and CDK4 in oral premalignant lesions and oral squamous cell carcinomas: a
In the present study, continuous p16 gene expression had a significant cell killing effect after irradiation, but transient expression had little effect. Establishment of a promising strategy for gene therapy treatment of human malignant glioma patients using irradiation plus p16 gene expression requires continuous transgene expression. The adenoviral vectors used here are effective for gene transfer to many tissues, and confer high levels of expression of recombinant genes. However, the use of firstgeneration vectors usually results in transient transgene expression only, because of the development of a cellular immune response triggered by viral proteins expressed by adenovirus genes, the dose of virus administered, the promoter chosen to drive expression of the recombinant gene, innate immune mechanisms and direct cytotoxicity caused by the expression of viral genes (Morral et al, 1999). Future studies of prolongation of in vivo expression of adenovirally administered transgenes with minimised direct cytotoxicity may clarify the number of functions mediated by p16 and p21 after irradiation (eg, modulation of cytokinesis or karyokinesis) and contribute to gene therapy treatment of human malignant glioma patients.

To confirm whether the effect of Ax-hp16 infection plus irradiation was specific for human glioma cell lines, we also performed the clonogenic assay for other p16-null human cancer cell lines infected with Ax-hp16: the non-small-cell lung cancer cell line A549 (p21, undetectable protein expression; p53, wild type), and the gastric cancer cell line MKN45 (p21 and p53, wild type). The results indicate that the cell survival of these cancer cell lines was substantially decreased by Ax-hp16 infection (data not shown) (Kawabe et al, 2000; Yokozaki, 2000). These findings suggest that adenovirus-mediated p16 gene transfer increased radiosensitivity via nonapoptotic cell killing in these other human cancer cell lines.

The present results demonstrate that p16 gene transfer following radiation results in cell death. The modulation of the cell death response by intervention in a specific signal transduction pathway between radiation and p16 in cytoplasm/membranes, perturbing cytokinesis and promoting abnormal nucleation, may enhance the cytotoxic effect of radiation, thus improving the therapeutic ratio.

\section{ACKNOWLEDGEMENTS}

We thank S Takeoka, K Kitagawa, S Suga, T Jo, A Okamoto, M Ueda, A Kamesako, M Nishimura, S Fujibuchi, K Fujiie, R Kadota and K Ogawa for technical assistance, and Drs H Yokozaki, A Noda and $\mathrm{T}$ Ishida for valuable discussion. This study was supported in part by grants-in-aid from the Ministry of Education, Science and Culture, Japan. semi-quantitative immunohistochemical study. J Oral Pathol Med 28: $158-164$

Cipriani B, Borsellino G, Knowles H, Tramonti D, Cavaliere F, Bernardi G, Battistini L, Brosnan, CF (2001) Curcumin inhibits activation of V9V2 T cells by phosphoantigens and induces apoptosis involving apoptosisinducing factor and large scale DNA fragmentation. J Immunol 167: $3454-3462$

Costanzi-Strauss E, Strauss BE, Naviaux RK, Haas M (1998) Restoration of growth arrest by p16INK4, p21WAF1, pRB, and p53 is dependent on the integrity of the endogenous cell-cycle control pathways in human glioblastoma cell lines. Exp Cell Res 238: 51-62

el-Deiry WS, Tokino T, Velculescu VE, Levy DB, Parsons R, Trent JM, Lin D, Mercer WE, Kinzler KW, Vogelstein B (1993) WAF1, a potential mediator of p53 tumour suppression. Cell 75: 817-825

Fueyo J, Gomez-Manzano C, Yung WK, Clayman GL, Liu TJ, Bruner J, Levin VA, Kyritsis AP (1996) Adenovirus-mediated p16/CDKN2 gene transfer induces growth arrest and modifies the transformed phenotype of glioma cells. Oncogene 12: 103-110 
Giangaspero F, Burger PC (1983) Correlations between cytologic composition and biologic behavior in the glioblastoma multiforme. A postmortem study of 50 cases. Cancer 52: 2320-2333

Gomez-Manzano C, Fueyo J, Kyritsis AP, Steck PA, Roth JA, McDonnell TJ, Steck KD, Levin VA, Yung WK (1996) Adenovirus-mediated transfer of the 53 gene produces rapid and generalized death of human glioma cells via apoptosis. Cancer Res 56: 694-699

Gomez-Manzano C, Fueyo J, Kyritsis AP, McDonnell TJ, Steck PA, Levin VA, Yung WK (1997) Characterization of p53 and p21 functional interactions in glioma cells en route to apoptosis. J Natl Cancer Inst 89: $1036-1044$

Hama S, Sadatomo T, Yoshioka H, Kurisu K, Tahara E, Naruse I, Heike Y, Saijo N (1997) Transformation of human glioma cell lines with the p16 gene inhibits cell proliferation. Anticancer Res 17: 1933-1938

Hama S, Heike Y, Naruse I, Takahashi M, Yoshioka H, Arita K, Kurisu K, Goldman CK, Curiel DT, Saijo N (1998) Adenovirus-mediated p16 gene transfer prevents drug-induced cell death through G1 arrest in human glioma cells. Int J Cancer 77: 47-54

Harada H, Nakagawa K, Iwata S, Saito M, Kumon Y, Sakaki S, Sato K, Hamada K (1999) Restoration of wild-type p16 down-regulates vascular endothelial growth factor expression and inhibits angiogenesis in human gliomas. Cancer Res 59: 3783-3789

He J, Allen JR, Collins VP, Allalunis-Turner MJ, Godbout R, Day RSIII, James CD (1994) CDK4 amplification is an alternative mechanism to p16 gene homozygous deletion in glioma cell lines. Cancer Res 54: $5804-5807$

Hwang HS, Davis TW, Houghton JA, Kinsella TJ (2000) Radiosensitivity of Thymidylate synthase-deficient human tumour cells is affected by progression through the G1 restriction point into S-phase: implications for fluoropyrimidine radiosensitization. Cancer Res 60: $92-100$

Kawabe S, Roth JA, Wilson DR, Meyn RE (2000) Adenovirus-mediated p16INK4a gene expression radiosensitizes non-small cell lung cancer cells in a p53-dependent manner. Oncogene 19: 5359-5366

Kawada M, Uehara Y, Mizuno S, Yamori T, Tsuruo T (1998) Up-regulation of p27Kip1 correlates inversely with anchorage-independent growth of human cancer cell lines. Jpn J Cancer Res 89: 110 - 115

Khodarev NN, Sokolova IA, Vaughan AT (1998) Mechanisms of induction of apoptotic DNA fragmentation. Int I Radiat Biol 73: 455-467

Li F, Ackermann EJ, Bennett CF, Rothermel AL, Plescia J, Tognin S, Villa A, Marchisio PC, Altieri DC (1999) Pleiotropic cell-division defects and apoptosis induced by interference with survivin function. Nat Cell Biol 1: $461-466$

Lukas J, Parry D, Aagaard L, Mann DJ, Bartkova J, Strauss M, Peters G, Bartek J (1995) Retinoblastoma-protein-dependent cell-cycle inhibition by the tumour suppressor p16. Nature 375: 503-506

Meyer-Puttlitz B, Hayashi Y, Waha A, Rollbrocker B, Bostrom J, Wiestler OD, Louis DN, Reifenberger G, von Deimling A (1997) Molecular genetic analysis of giant cell glioblastomas. Am J Pathol 151: 853-857

Miller CR, Buchsbaum DJ, Reynolds PN, Douglas JT, Gillespie GY, Mayo MS, Raben D, Curiel DT (1998) Differential susceptibility of primary and established human glioma cells to adenovirus infection: targeting via the epidermal growth factor receptor achieves fiber receptor-independent gene transfer. Cancer Res 58: 5738-5748

Milligan A, Gabrielli BG, Clark JM, Hayward NK, Ellem KA (1998) Involvement of p16CDKN2A in cell-cycle delays after low dose UV irradiation. Mutat Res 422: 43-53

Miyake S, Makimura M, Kanegae Y, Harada S, Sato Y, Takamori K, Tokuda C, Saito I (1996) Efficient generation of recombinant adenoviruses using adenovirus DNA-terminal protein complex and a cosmid bearing the full-length virus genome. Proc Natl Acad Sci USA 93: $1320-1324$

Morral N, O'Neal W, Rice K, Leland M, Kaplan J, Piedra PA, Zhou H, Parks RJ, Velji R, Aguilar-Cordova E, Wadsworth S, Graham FL, Kochanek S, Carey KD, Beaud AL (1999) Administration of helper-dependent adenoviral vectors and sequential delivery of different vector serotype for long-term liver-directed gene transfer in baboons. Proc Natl Acad Sci USA 96: $12816-12821$
Niwa H, Yamamura K, Miyazaki J (1991) Efficient selection for highexpression transfectants with a novel eukaryotic vector. Gene 108: $193-199$

Nobori T, Miura K, Wu DJ, Lois A, Takabayashi K, Carson DA (1994) Deletions of the cyclin-dependent kinase-4 inhibitor gene in multiple human cancers. Nature 368: 753-756

Noda A, Ning Y, Venable SF, Pereira-Smith OM, Smith JR (1994) Cloning of senescent cell-derived inhibitors of DNA synthesis using an expression screen. Exp Cell Res 211: $90-98$

Okada S (1970) Variation in radiation response with growth phase or cellcycle stage. In Radiation Biochemistry, Altman KI, Cerber GB, Okada S. (eds) Vol. I pp 146-163. London, LTD: Academic Press

Okahashi N, Murase Y, Koseki T, Sato T, Yamato K, Nishihara T (2001) Osteoclast differentiation is associated with transient upregulation of cyclin-dependent kinase inhibitors p21(WAF1/CIP1) and p27(KIP1). $J$ Cell Biochem 80: $339-345$

Ostruszka LJ, Shewach DS (2000) The role of cell-cycle progression in radiosensitization by $2^{\prime}, 2^{\prime}$-difluoro-2'-deoxycytidine. Cancer Res 60: $6080-6088$

Pavey S, Conroy S, Russell T, Gabrielli B (1999) Ultraviolet radiation induces p16CDKN2A expression in human skin. Cancer Res 59: $4185-4189$

Sandig V, Brand K, Herwig S, Lukas J, Bartek J, Strauss M (1997) Adenovirally transferred p16INK4/CDKN2 and p53 genes cooperate to induce apoptotic tumour cell death. Nat Med 3: 313-319

Sano T, Oyama T, Kashiwabara K, Fukuda T, Nakajima T (1998) Immunohistochemical overexpression of p16 protein associated with intact retinoblastoma protein expression in cervical cancer and cervical intraepithelial neoplasia. Pathol Int 48: 580-585

Schmidt EE, Ichimura K, Reifenberger G, Collins VP (1994) CDKN2 (p16/ MTS1) gene deletion or CDK4 amplification occurs in the majority of glioblastomas. Cancer Res 54: 6321-6324

Sherr CJ (1996) Cancer cell cycles. Science 274: $1672-1677$

Tanaka Y, Maruo A, Fujii K, Nomi M, Nakamura T, Eto S, Minami Y (2000) Intercellular adhesion molecule 1 discriminates functionally different populations of human osteoblasts: characteristic involvement of cellcycle regulators. J Bone Miner Res 15: $1912-1923$

Tang DC, Jennelle RS, Shi Z, Garver Jr RI, Carbone DP, Loya F, Chang CH, Curiel DT (1997) Overexpression of adenovirus-encoded transgenes from the cytomegalovirus immediate early promoter in irradiated tumour cells. Hum Gene Ther 8: 2117-2124

Tauchi H, Kobayashi J, Morishima K, Matsuura S, Nakamura A, Shiraishi T, Ito E, Masnada D, Delia D, Komatsu K (2001) The forkhead-associated domain of NBS1 is essential for nuclear foci formation after irradiation but not essential for hRAD50-hMRE11-NBS1 complex DNA repair activity. J Biol Chem 276: $12-15$

Teramoto S, Johnson LG, Huang W, Leigh MW, Boucher RC (1995) Effect of adenoviral vector infection on cell proliferation in cultured primary human airway epithelial cells. Hum Gene Ther 6: $1045-1053$

Terasima T, Tolmach LJ (1963) Variations in several responses of HeLa cells to $\mathrm{x}$-irradiation during the division cycle. Biophysical J 3: 11-33

Ueki K, Ono Y, Henson JW, Efird JT, von Deimling A, Louis DN (1996) CDKN2/p16 or RB alterations occur in the majority of glioblastomas and are inversely correlated. Cancer Res 56: $150-153$

Wang XQ, Gabrielli BG, Milligan A, Dickinson JL, Antalis TM, Ellem KA (1996) Accumulation of p16CDKN2A in response to ultraviolet irradiation correlates with late S-G(2)-phase cell cycle delay. Cancer Res 56: $2510-2514$

Xiong Y, Hannon GJ, Zhang H, Casso D, Kobayashi R, Beach D (1993) p21 is a universal inhibitor of cyclin kinases. Nature 366: 701-704

Yamasaki F, Hama S, Yoshioka H, Kajiwara Y, Yahara K, Sugiyama K, Heike Y, Arita K, Kurisu K (2003) Staurosporine-induced apoptosis is independent of p16 and p21 and achieved via arrest at G2/M and at G1 in U251MG human glioma cell line. Cancer Chemother Pharmacol 51: $271-283$

Yokozaki H (2000) Molecular characteristics of eight gastric cancer cell lines established in Japan. Pathol Int 50: 767-777 